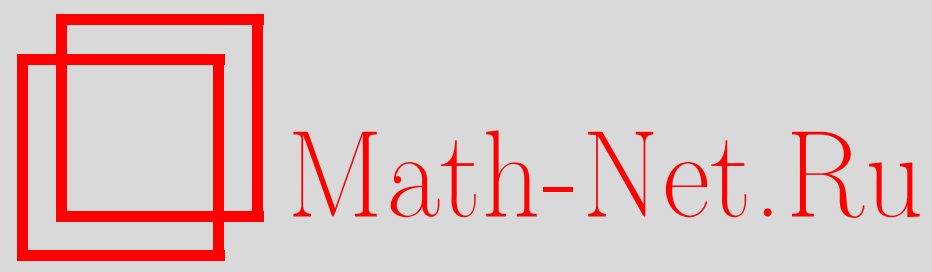

С. П. Струнков, О слабо коспектральных графах, Матем. заметки, 2004, том 75, выпуск 4, 614-623

DOI: https://doi.org/10.4213/mzm57

Использование Общероссийского математического портала Math-Net.Ru подразумевает, что вы прочитали и согласны с пользовательским соглашением http://www .mathnet.ru/rus/agreement

Параметры загрузки:

IP : 52.90 .164 .192

26 апреля 2023 г., 17:20:25

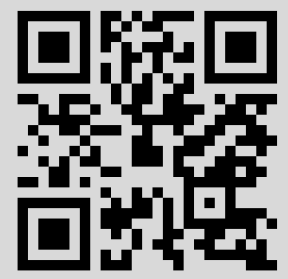




\title{
О СЛАБО КОСПЕКТРАЛЬНЫХ ГРАФАХ
}

\author{
С. П. Струнков
}

\begin{abstract}
Доказывается конечность любого множества конечных попарно неизоморфных слабо коспектральных связных псевдографов, а также псевдоорграфов, удовлетворяющих различным дополнительньм условиям.

Библиография: 8 названий.
\end{abstract}

Вопросы о том, в какой мере информация о различных свойствах и параметрах спектра графа дает возможность восстановить граф, относятся к числу важнейших в спектральной теории графов. Заметим, что даже по характеристическому многочлену матрицы смежности графа сам граф не всегда определяется однозначно; к настоящему времени найдено много пар коспектральных графов [1]. Поэтому при изучении множества неизоморфных графов, спектры которых обладают некоторыми заданньми свойствами, вопрос о конечности этого множества представляет интерес в первую очередь. Такого рода вопросы о конечности числа алгебраических или комбинаторных объектов, обладающих заданными свойствами, в последнее время все чаще называют проблемами бернсайдовского типа. Имя этому направлению исследований дала ослабленная проблема Бернсайда (ОПБ) о конечности числа неизоморфных конечных групп с заданными числом образующих и показателем [2]. Так как число образуюших конечной групшы относится к числу спектральных характеристик ее графа Кэли, то это обстоятельство сближает рассматриваемые вопросы для графов с ОПБ.

В настоящей работе рассматриваются конечные псевдографы и псевдоорграфы [3], т.е. неориентированные и ориентированные конечные графы, в которых допускается наличие кратных ребер и петель. Мы и дальше будем придерживаться терминологии, принятой в [3]. Два псевдографа (или псевдоорграфа) $G_{1}$ и $G_{2}$ будем называть здесь слабо коспектральныции, если каждое характеристическое число матрицы смежности $M_{G_{1}}$ псевдографа (псевдоорграфа) $G_{1}$ является характеристическим числом матрицы смежности $M_{G_{2}}$ псевдографа (псевдоорграфа) $G_{2}$ и наоборот. Слабо коспектральные псевдографы и псевдоорграфы в отличие от коспектральньх могут иметь разные количества вершин. Поэтому вопрос о том, насколько широкими являются различные классы слабо коспектральных псевдографов и псевдоорграфов, в какой мере определяюшим является свойство слабой коспектральности, представляется естественным. Этот вопрос оказался тесно связанным с аналогичньми вопросами для других параметров спектра графа, например, для числа попарно различных характеристических

Работа поддержана Российским фондом фундаментальных исследований, грант № 02-01-00581. 
чисел графа при дополнительных ограничениях для главного из них. Первый результат в решении подобных проблем был получен Цветковичем. Им была доказана конечность числа связных регулярных графов заданной валентности с цельп рациональньм спектром [4], [1]. В настоящей работе эти вопросы рассматриваются для различных классов псевдографов и псевдоорграфов, при этом в теореме 6 значительно усиливается этот результат Цветковича.

Понятно, что операция объединения нескольких слабо коспектральных, в частности, изоморфных, псевдографов или псевдоорграфов позволяет строить бесконечные множества попарно неизоморфных слабо коспектральных псевдографов и псевдоорграфов, т.е. может показаться, что классы слабо коспектральных псевдографов и псевдоорграфов необозримы. Однако для псевдографов эта конструкция является в некотором смысле единственным средством построения бесконечных множеств неизоморфных слабо коспектральных псевдографов, как показьвает следующая

ТЕорема 1. Для любого вещественного числа $r$ и натурального $k$ мнохсество конечных неизоморфных связных псевдографов, матрицы смехнности которых имеют $k$ попарно различных характеристических чисел, максимальное из которых не превосходит $r$, конечно.

ДокАЗАТЕЛЬСТво. Пусть $\lambda_{1}>\lambda_{2}>\cdots>\lambda_{k}-$ множество попарно различных характеристических чисел матрищы смежности $M_{G}=\left(a_{i j}\right)$ некоторого связного конечного псевдографа $G$, имеющего $n>1$ вершин. Так как все элементы матрицы $M_{G}$ неотрицательны, то по теореме $\Phi$ робениуса [5, глава 8] $\lambda_{1}>0$ и $\left|\lambda_{\nu}\right| \leqslant \lambda_{1} \leqslant r$ для любого $\nu=2, \ldots, k$. Установим теперь, что для любого $i=1, \ldots, n$ степень $V_{i} i$-ой вершины псевдографа $G$ не превосходит числа $r^{2}+r$. Понятно, что $a_{i i} \leqslant r$. Покажем, что число

$$
\sigma_{i}=\sqrt{\sum_{\substack{j=1 \\ j \neq i}}^{n} a_{i j}^{2}} \leqslant r .
$$

Для этого рассмотрим квадратичную форму $q(x)$ в $n$-мерном евклидовом пространстве $\mathbf{E}$, матрица которой в некотором ортонормированном базисе $e_{1}, \ldots, e_{n}$ совпадает с матрицей $M_{G}$. Тогда сужение $q(x)$ на двумерное подпространство $\mathbf{E}^{\prime}$, натянутое на ортонормированную систему векторов

$$
e_{i}, \quad e_{i}^{\prime}=\frac{a_{i 1}}{\sigma_{i}} e_{1}+\cdots+\frac{a_{i i-1}}{\sigma_{i}} e_{i-1}+\frac{a_{i i+1}}{\sigma_{i}} e_{i+1}+\cdots+\frac{a_{i n}}{\sigma_{i}} e_{n},
$$

имеет в этом базисе матрищу

$$
M^{\prime}=\left(\begin{array}{cc}
a_{i i} & \sigma_{i} \\
\sigma_{i} & b
\end{array}\right),
$$

где $b=q\left(e_{i}^{\prime}\right)$. Легко проверить, что разность характеристических чисел $\lambda_{1}^{\prime}>\lambda_{2}^{\prime}$ матрицы $M^{\prime}$

$$
\lambda_{1}^{\prime}-\lambda_{2}^{\prime}=\sqrt{\left(a_{i i}-b\right)^{2}+4 \sigma_{i}^{2}} \geqslant 2 \sigma_{i} .
$$

Так как значения квадратичной формы на единичной сфере заключаются между наименьшим и наибольшим из характеристических чисел ее матрищы в соответствующем ортонормированном базисе, то

$$
2 \sigma_{i} \leqslant \lambda_{1}^{\prime}-\lambda_{2}^{\prime} \leqslant \lambda_{1}-\lambda_{k} \leqslant 2 r .
$$


Следовательно,

$$
V_{i}=\sum_{j=1}^{n} a_{i j} \leqslant a_{i i}+\sigma_{i}^{2} \leqslant r+r^{2}
$$

Так как $G$ - связньй псевдограф, то его матрица смежности $M_{G}$ неразложима, симметрична и, как уже говорилось выше, все ее элементы неотрицательны. Поэтому для любых несовпадающих индексов $i, j \in\{1,2, \ldots, n\}$ найдется такое натуральное число $\delta$, для которого $(i j)$-ьй элемент матрицы $M_{G}^{\delta}$ отличен от нуля. Минимальное среди всех таких чисел для данных индексов $i, j$ обозначим через $\delta^{(i j)}$, а через $d$ обозначим максимальное из всех чисел $\delta^{(i j)}$. Легко проверить, что для любого натурального числа $m$ сумма элементов каждой строки матрицы $M_{G}^{m}$ не превосходит числа $\left(r^{2}+r\right)^{m}$, следовательно,

$$
1+\left(r^{2}+r\right)+\left(r^{2}+r\right)^{2}+\cdots+\left(r^{2}+r\right)^{d} \geqslant n
$$

откуда получаем, что

$$
n \leqslant \frac{\left(r^{2}+r\right)^{d+1}-1}{r^{2}+r-1}
$$

Пусть $P(t)=t^{k}+\alpha_{1} t^{k-1}+\cdots+\alpha_{k}$ - минимальньй многочлен матрищы $M_{G}$. Его степень $k$ равна числу попарно различных характеристических чисел матрищы $M_{G}$. Если $d \geqslant k$, то

$M_{G}^{d-k} P\left(M_{G}\right)=M_{G}^{d-k}\left(M_{G}^{k}+\alpha_{1} M_{G}^{k-1}+\cdots+\alpha_{k} E\right)=M_{G}^{d}+\alpha_{1} M_{G}^{d-1}+\cdots+\alpha_{k} M_{G}^{d-k}=0$.

Но последнее равенство невозможно ввиду того, что $(i j)$-ьй элемент в левой части, для которого $d=\delta^{(i j)}$, не равен нулю. Следовательно, $d<k$ и

$$
n \leqslant \frac{\left(r^{2}+r\right)^{k+1}-1}{r^{2}+r-1} .
$$

Утверждение теоремы вытекает из последнего неравенства и установленных выше оцеHOK

$$
0 \leqslant a_{i i} \leqslant r, \quad 0 \leqslant a_{i j} \leqslant \sigma_{i} \leqslant r, \quad i \neq j
$$

для коэффициентов матрицы $M_{G}$.

СЛЕДСТВИЕ. Любое множество конечных попарно неизоморфных слабо коспектральных связных псевдографов конечно.

Однако, даже для сильно связных регулярных орграфов утверждения теоремы 1 и ее следствия перестают быть верными. Опишем соответствующий контрпример. Для заданного псевдоорграфа $G$ обозначим через $L(G)$ его реберны й псевдоорграф, т.е. псевдоорграф, вершинами которого являются все дуги псевдоорграфа $G$, а дугами - всевозможные упорядоченные пары вершин в $L(G)$ вида $\left((u, v)_{i},(v, w)_{j}\right)$. Эта конструкция является аналогом соответствующего определения реберного графа для неориентированных графов [3], [1], но спектральные свойства ориентированных и неориентированных реберных графов различны. 
ТЕорема 2. Множества ненулевых характеристических чисел матрии смежности $M_{G}$ и $M_{L(G)}$ псевдоорграфов $G$ и $L(G)$ совпадают. При этом кратности каждого ненулевого корня в характеристических уравнениях матрии, $M_{G} u M_{L(G)}$ равнbl.

ДокАЗАТЕЛЬСТво. Пусть $v_{1}, v_{2}, \ldots, v_{n}$ - все вершины и $\varepsilon_{1}, \ldots, \varepsilon_{m}-$ все дуги псевдоорграфа $G$. По определению матрищы смежности $M_{G}=\left(a_{i j}\right)$ псевдоорграфа $G$ коэффициент $a_{i j}$ равен числу ориентированных ребер, идуших из $i$-ой вершины в $j$-ую. $M a m$ рицей исходов псевдоорграфа $G$ будем называть матрицу $S_{G}=\left(s_{i k}\right)$ размера $n \times m$, $(i k)$-ый элемент $s_{i k}$ которой равен единице, если дуга $\varepsilon_{k}$ псевдоорграфа $G$ выходит из вершины $v_{i}$, и нулю - в противном случае. Аналогично матрицей заходов псевдоорграфа $G$ назовем матрищу $F_{G}=\left(f_{k j}\right)$ размера $m \times n,(k j)$-ьй элемент $f_{k j}$ которой равен единице, если конец дуги $\varepsilon_{k}$ находится в вершине $v_{j}$, и нулю, если это не так. Пусть $\Sigma(G)$ - псевдоорграф подразбиения псевдоорграфа $G$, определяемьй естественным образом как псевдоорграф с вершинами $u_{1}, \ldots, u_{n}, u_{1+n}, \ldots, u_{m+n}$, ориентированньми ребрами которого являются такие упорядоченные пары

$$
\left(u_{i}, u_{k+n}\right), \quad\left(u_{k+n}, u_{j}\right), \quad i, j \in\{1,2, \ldots, n\}, \quad k \in\{1,2, \ldots, m\},
$$

его вершин, для соответствующих индексов $i, j, k$ которых в псевдоорграфе $G$ упорядоченное ребро $\varepsilon_{k}$ исходит из вершины $v_{i}$, а заходит в вершину $v_{j}$. Легко проверить, что

$$
M_{\Sigma(G)}=\left(\begin{array}{cc}
0 & S_{G} \\
F_{G} & 0
\end{array}\right), \quad M_{\Sigma(G)}^{2}=\left(\begin{array}{cc}
M_{G} & 0 \\
0 & M_{L(G)}
\end{array}\right)
$$

откуда получаем, что

$$
M_{G}=S_{G} F_{G}, \quad M_{L(G)}=F_{G} S_{G}
$$

Утверждение теоремы теперь вытекает из свойств характеристических многочленов матриц $A B$ и $B A$, где $A$ и $B$ - произвольные матрицы размеров $n \times m$ и $m \times n$ соответственно [6, п. 2.15.15].

Если $G$ - произвольный конечньй сильно связньй регулярньй псевдоорграф степени $r$ (т.е. полустепени исхода и захода каждой вершины в $G$ равны числу $r$ ), то $L(G)$ является также сильно связным регулярным псевдоорграфом степени $r$ и при этом $|L(G)|$ $=|G|_{r}$ (через $|G|$ мы обозначаем здесь число вершин псевдоорграфа $G$ ). Если псевдоорграф $G$ не имеет петель (т.е. $G$ - мультиорграф), то $L(G)$ является орграфом (т.е. не имеет петель и кратных дуг). Контрпример к теореме 1 и ее следствию для регулярных сильно связных орграфов получается при $r \geqslant 2$; в этом случае все орграфы

$$
L(G), \quad L^{2}(G)=L(L(G)), \quad \ldots, \quad L^{k}(G)=L\left(L^{k-1} G\right), \quad \ldots
$$

попарно неизоморфны, сильно связны и слабо коспектральны для любого конечного сильно связного регулярного мультиграфа $G$. Аналогичным образом можно построить разнообразные множества (в том числе бесконечные) слабо коспектральных псевдоорграфов и с помощю других псевдоорграфов (например, не обязательно регулярных). Можно ожидать, что эти множества могут оказаться чересчур широкими. Поэтому нашей дальнейшей целью будет изучение общих свойств регулярных слабо коспектральных сильно связных псевдоорграфов. Мы покажем, что этот класс псевдоорграфов наиболее близок к псевдографам. 
Заметим, что в приведенном вьше примере последовательности псевдоорграфов $L(G)$, $L^{2}(G), \ldots, L^{k}(G), \ldots$, построенной по регулярному псевдоорграфу $G$, объединение простых делителей всех чисел $\left|L^{k}(G)\right|$ конечно. Покажем, что это свойство является типичным для всех множеств регулярных сильно связных слабо коспектральных псевдоорграфов. Предположим, что $G$ - конечный псевдоорграф и число его вершин

$$
|G|=p_{1}^{h_{1}} p_{2}^{h_{2}} \cdots p_{l}^{h_{l}}
$$

где $p_{1}, \ldots, p_{l}$ - простые числа, а $h_{1}, \ldots, h_{l}$ - целые положительные. Число $\rho(G)$ $=p_{1} p_{2} \cdots p_{l}$ будем называть арифметической длиной псевдоорграфа $G$, а максимальное из чисел $h_{1}, \ldots, h_{l}$ - его арифметической высотой.

ТЕОрема 3. Множество арифметических длин любого множсества конечных слабо коспектральных сильно связных регулярных псевдоорграфов конечно.

ДокАЗАТЕЛЬСТВО. Предположим, что $G$ - некоторый конечньй сильно связньй регулярньй псевдоорграф степени $r$ и $\lambda_{1}=r, \lambda_{2}, \ldots, \lambda_{k}$ - все попарно различные характеристические числа его матрицы смежности $M_{G}, c_{i}$ - максимум порядков жордановых клеток жордановой формы матрищы $M_{G}$ с числом $\lambda_{i}$ на диагонали. Покажем вначале, что число

$$
b=\prod_{i=2}^{k}\left(r-\lambda_{i}\right)^{c_{i}}
$$

является цельм рациональным и при этом делится на число $n=|G|$ вершин псевдоорграфа $G$.

Действительно, так как матрища $M_{G}$ кратна двояко стохастической матрище, а также является неразложимой матрицей с неотрицательными элементами (это вытекает из сильной связности псевдоорграфа $G)$, то $c_{1}=1[5$, глава 8]. Отсюда легко вытекает, что матрища

$$
B=\prod_{i=2}^{k}\left(M_{G}-\lambda_{i} E\right)^{c_{i}}=M_{G}^{m}+\alpha_{1} M_{G}^{m-1}+\cdots+\alpha_{m} E,
$$

где $E$ - единичная матрища и $m=c_{2}+\cdots+c_{k}$, имеет ранг 1 . Легко проверить, что для любого натурального числа $i$ матрица $M_{G}^{i}$ также кратна двояко стохастической матрице и сумма элементов каждой ее строки и каждого столбца равна $r^{i}$. Поэтому сумма элементов каждой строки и каждого столбца матрицы $B$ равна

$$
r^{m}+\alpha_{1} r^{m-1}+\cdots+\alpha_{m}=\prod_{i=2}^{k}\left(r-\lambda_{i}\right)^{c_{i}},
$$

т.е. отлична от нуля. Следовательно, все элементы матрищы $B$ равны между собой. Так как все элементы матриц $M_{G}^{i}$ и числа $\alpha_{i}$ являются целыми рациональными, то элементы матрицы $B$ - также целые рациональные числа. Поэтому след $\operatorname{tr}(B)$ матрицы $B$ является цельм рациональным числом и делится на $n$. С другой стороны, если вычислять матрицу $B$, используя жорданову форму матрицы $M_{G}$, то получим, что $B$ подобна матрице, имеющей единственньй ненулевой элемент

$$
b=\prod_{i=2}^{k}\left(r-\lambda_{i}\right)^{c_{i}}
$$


стоящий на главной диагонали, т.е. $\operatorname{tr}(B)=b$. Таким образом, число $b$ является целым рациональньм и при этом делится на $n$.

Покажем теперь, что число

$$
b_{1}=\prod_{i=2}^{k}\left(r-\lambda_{i}\right)
$$

также является цельм рациональным и при этом делится на арифметическую длину $\rho(G)$ рассматриваемого псевдоорграфа $G$. Действительно, так как $\lambda_{1}$ - целое рациональное число, то множество чисел $\lambda_{2}, \ldots, \lambda_{k}$ совпадает с объединением орбит группы Галуа характеристического многочлена матрицы $M_{G}$, имеющего целые рациональные коэффициенты и единицу при старшей степени его аргумента. Следовательно, число $b_{1}$ является цельм рациональньм. С другой стороны, легко показать, что некоторая степень $b_{1}^{c}$ числа $b_{1}$ (например, при $c=\max \left\{c_{2}, \ldots, c_{k}\right\}$ ) делится на число $n=|G|$. Поэтому число $b_{1}$ делится на $\rho(G)$. Следовательно,

$$
\rho(G) \leqslant \prod_{i=2}^{k}\left(r-\lambda_{i}\right)
$$

и теорема доказана.

СЛЕДСТВИЕ. Для любого числа $H$ множсество конечных попарно неизоморфных слабо коспектральных регулярных сильно связных псевдоорграфов, арифметические высоты которых не превосходят числа $H$, конечно.

Естественно назьвать число $c=\max \left\{c_{2}, \ldots, c_{k}\right\}$ (т.е. индекс нильпотентности матрицы $M_{G}$ ) индексом нильпотентности конечного псевдоорграфа $G$. Из неравенства

$$
|G| \leqslant \prod_{i=2}^{k}\left(r-\lambda_{i}\right)^{c_{i}},
$$

доказанного в предыдущей теореме, вытекает конечность любого множества попарно неизоморфных слабо коспектральных сильно связных регулярных псевдоорграфов, множество индексов нильпотентности которых ограничено сверху. Так как $r=\lambda_{1}$ - максимальное характеристическое число неотрищательной матрицы $M_{G}$, то $\left|\lambda_{i}\right| \leqslant r$ для всех $i=2, \ldots, k$. Поэтому из предыдущего неравенства получаем, что

$$
|G| \leqslant(2 r)^{c(k-1)} .
$$

При исследовании алгебраических и комбинаторных объектов часто возникают различные классы псевдоорграфов. В частности, графы Кэли, встречаемыев теории конечных групп, дают типичные примеры таких псевдоорграфов. Они регулярны, их степень равна числу образующих группы, по которым они построены. Характерным свойством графа Кэли является его вершинная симметричность, т.е. транзитивность на вершинах его групшы автоморфизмов. Справедливо следующее общее утверждение для таких псевдоорграфов.

ТЕОремА 4. Любое множсество конечных попарно неизоморфных слабо коспектральных сильно связных вериинно-симметричных псевдоорграфов конечно. 
ДоКАЗАТЕЛЬСтво. Пусть $G$ - конечньй сильно связный вершинно-симметричньй псевдоорграф степени $r$, имеющий $n>1$ вершин. Тогда его матрища смежности $M_{G}$ является неотрицательной, неразложимой матрищей порядка $n$ с целыми рациональньми элементами. Кроме того, $M_{G}$ кратна двояко стохастической матрище и сумма элементов каждой ее строки и каждого столбца равна $r$. Легко проверить, что для любого натурального числа $i$ матрища $M_{G}^{i}$ обладает этими же свойствами и при этом сумма элементов каждой ее строки и каждого столбца равна $r^{i}$.

Обозначим через $\lambda_{1}=r, \lambda_{2}, \ldots, \lambda_{k}$ все попарно различные характеристические числа матрицы $M_{G}$, а через $s_{i}$ - кратность $\lambda_{i}$ как корня характеристического уравнения матрицы $M_{G}$. Тогда $s_{1}=1$, а также

$$
\sum_{i=1}^{k} s_{i}=n
$$

Если $\nu$ - целое рациональное положительное число, то для $\nu>1$

$$
\operatorname{tr}\left(M_{G}^{\nu-1}\right)=\sum_{i=1}^{k} s_{i} \lambda_{i}^{\nu-1}
$$

Так как все диагональные элементы каждой матрицы $M_{G}^{\nu-1}$ равны между собой, то обозначив их общее значение через $\beta_{\nu}$, получаем следующую систему равенств

$$
\sum_{i=1}^{k} s_{i} \lambda_{i}^{\nu-1}=\beta_{\nu} n, \quad \nu=2, \ldots, k
$$

Из системы равенств (1), (2) получаем

$$
s_{i}=\frac{W_{i}}{W\left(\lambda_{1}, \ldots, \lambda_{k}\right)}, \quad i=1, \ldots, k,
$$

где $W\left(\lambda_{1}, \ldots, \lambda_{k}\right)$ - определитель Вандермонда, построенньй по системе чисел $\lambda_{1}, \ldots$, $\lambda_{k}$, a $W_{i}$ - определитель, полученный из определителя $W\left(\lambda_{1}, \ldots, \lambda_{k}\right)$ заменой $i$-ого столбца на столбец, состоящий из чисел $n, \beta_{2} n, \ldots, \beta_{k} n$. Процесс разложения определителя $W_{i}$ по $i$-ому столбцу приводит к равенствам

$$
W_{i}=n \sum_{j=1}^{k}(-1)^{j+i} \beta_{j} W_{i j}, \quad i=1, \ldots, k,
$$

в которых $\beta_{1}=1, W_{i j}$ - определитель, полученньй из $W\left(\lambda_{1}, \ldots, \lambda_{k}\right)$ вычеркиванием $i$-го столбца и $j$-ой строки. Как известно,

$$
W_{i j}=S_{k-j}\left(\lambda_{1}, \ldots, \lambda_{i-1}, \lambda_{i+1}, \ldots, \lambda_{k}\right) W\left(\lambda_{1}, \ldots, \lambda_{i-1}, \lambda_{i+1}, \ldots, \lambda_{k}\right)
$$

(в этих равенствах через $S_{k}\left(x_{1}, \ldots, x_{l}\right)$ обозначен $k$-ьй элементарньй симметрический многочлен от $l$ аргументов $\left.\left(x_{1}, \ldots, x_{l}\right)\right)$. После сокращения одинаковых сомножителей, входящих в выражение для $s_{i}$ от $W\left(\lambda_{1}, \ldots, \lambda_{k}\right)$ и $W\left(\lambda_{1}, \ldots, \lambda_{i-1}, \lambda_{i+1}, \ldots, \lambda_{k}\right)$, получим

$$
s_{i}=\frac{n \sum_{j=1}^{k}(-1)^{j+i} \beta_{j} S_{k-j}\left(\lambda_{1}, \ldots, \lambda_{i-1}, \lambda_{i+1}, \ldots, \lambda_{k}\right)}{\prod_{\substack{j=1 \\(j \neq i)}}^{k}\left(\lambda_{i}-\lambda_{j}\right)} .
$$


Tак как $s_{1}=1, \lambda_{1}=r$, то

$$
\prod_{j=2}^{k}\left(r-\lambda_{j}\right)=n \sum_{j=1}^{k}(-1)^{j+1} \beta_{j} S_{k-j}\left(\lambda_{2}, \ldots, \lambda_{k}\right) .
$$

Легко видеть, что все числа $\prod_{j=2}^{k}\left(r-\lambda_{j}\right), S_{k-j}\left(\lambda_{2}, \ldots, \lambda_{k}\right)$ являются цельми рациональными. Следовательно,

$$
\prod_{j=2}^{k}\left(r-\lambda_{j}\right) \equiv 0 \quad(\bmod n)
$$

откуда получаем оценку

$$
n \leqslant \prod_{j=2}^{k}\left(r-\lambda_{j}\right) .
$$

Так как сумма элементов в каждой строке матрищы $M_{G}$ равна $r=\lambda_{1}$ и элементы этой матрицы - целые неотрицательные числа, то для любых индексов $i, j \in\{1,2, \ldots, n\}$ выполняется неравенство $a_{i j} \leqslant r$. Из последних двух неравенств для $n$ и $a_{i j}$ вытекает утверждение теоремы.

Как и в предыдущей теореме, легко получить оценку

$$
n \leqslant(2 r)^{k-1}
$$

для числа вершин $n$ сильно связного вершинно-симметричного псевдоорграфа $G$ степени $r$, матрица смежности которого имеет $k$ попарно различных характеристических чисел.

Еще более слабым отношением эквивалентности для псевдографов и псевдоорграфов, чем слабая коспектральность, является эквивалентность, основанная на сравнении алгебраических степеней чисел из их спектров. Числовым параметром псевдографа или псевдоорграфа $G$, с помощью которого строится это отношение эквивалентности, является его алгебраическая степень, которую определим здесь как максимум алгебраических степеней характеристических чисел его матрицы смежности $M_{G}$. Покажем, что слабая коспектральность часто является не намного более сильньм ограничением, чем ограничение на алгебраическую степень псевдографов и псевдоорграфов. Назовем степенью псевдографа максимум степеней его вершин, а полустепенью исхода $r_{s}(G)$ ( захода $\left.r_{f}(G)\right)$ псевдоорграфа максимум полустепеней исхода (соответственно захода) каждой его вершины. Как обычно, степень вершины $v$ псевдографа $G$ определяется как число ребер, инцидентных вершине $v$ (при этом каждая петля считается два раза), а если $G$ - псевдоорграф, то полустепень исхода $r_{s}(v)$ его вершины $v$ - это число дуг, начинающихся в $v$. Аналогично определяется полустепень захода $r_{f}(v)$ произвольной вершины $v$ псевдоорграфа $G$. Справедливо следующее утверждение.

ТЕОРемА 5. Пусть $r$ и $m$ - произвольные натуральные числа, $\Sigma$ - некоторое множество конечных псевдографов и псевдоорграфов, удовлетворяюших следующим условиям:

1) алгебраическая степень каждого псевдографа и псевдоорграфа $G \in \Sigma$ не превосходит числа $\mathrm{m}$;

2) степень каждого псевдографа, а также минимальное из чисел $r_{s}(G), r_{f}(G)$ для каждого псевдоорграфа $G \in \Sigma$ не больше числа $r$. 
Тогда число различных классов попарно слабо коспектральных псевдографов $и$ псевдоорграфов, содерэсащихся в $\Sigma$, конечно.

ДокАЗАТЕЛЬСТво. Предположим, что $G$ - псевдограф или псевдоорграф, принадлежащий множеству $\Sigma$ и имеющий $n>1$ вершин. Если $\lambda$-характеристическое число его матрицы смежности $M_{G}$, то по теореме Гершгорина $[5$, глава $6, \S 5]$ для некоторых индексов $i, j \in\{1, \ldots, n\}$ выполняются неравенства

$$
\left|\lambda-a_{i i}\right| \leqslant \sum_{\substack{s=1 \\ s \neq i}}^{n}\left|a_{i s}\right|, \quad\left|\lambda-a_{j j}\right| \leqslant \sum_{\substack{s=1 \\ s \neq j}}^{n}\left|a_{s j}\right|,
$$

откуда в силу наших условий получаем, что $|\lambda| \leqslant r$. Пусть $\lambda_{1}=\lambda, \lambda_{2}, \ldots, \lambda_{k}-$ все корни неприводимого над полем $\mathbb{Q}$ рациональных чисел многочлена с целыми рациональньми коэффициентами, корнем которого является число $\lambda$. Так как коэффициенты характеристического многочлена матрицы $M_{G}$ являются целыми рациональньми числами, то все числа $\lambda_{i}, i=1, \ldots, k$, также принадлежат спектру матрищы $M_{G}$ и, следовательно, $\left|\lambda_{i}\right| \leqslant r$ для всех $i=1, \ldots, k$ и $k \leqslant m$. Рассмотрим многочлен

$$
P(t)=\left(t-\lambda_{1}\right)\left(t-\lambda_{2}\right) \cdots\left(t-\lambda_{k}\right)=t^{k}+\alpha_{1} t^{k-1}+\cdots+\alpha_{k} .
$$

Коэффициенты $\alpha_{i}$ этого многочлена являются целыми рациональными числами. Из выражений $\alpha_{i}$ через $\lambda_{1}, \ldots, \lambda_{k}$ и неравенств $\left|\lambda_{i}\right| \leqslant r$ получаем, что для любого $i=1, \ldots, k$

$$
\left|\alpha_{i}\right| \leqslant\left(\begin{array}{c}
k \\
i
\end{array}\right) r^{i} \leqslant m ! r^{m} .
$$

Таким образом, различных многочленов $P(t)$, корнями которых являются характеристические числа матриц смежности псевдографов и псевдоорграфов, принадлежащих множеству $\Sigma$, может сушествовать лиш конечное число и, следовательно, количество попарно различных чисел $\lambda$, каждое из которых принадлежит спектру матрицы $M_{G}$ для какого-нибудь псевдографа или псевдоорграфа $G \in \Sigma$, ограничено сверху некоторьм конечньм числом, зависящим только от чисел $r$ и $m$. Теорема, тем самьм, полностью доказана.

Из цитированной вьше теоремы Гершгорина, вытекает, что максимальное характеристическое число (назьваемое главным) матрицы смежности псевдоорграфа не превосходит минимума его полустепеней исхода и захода. Так как в доказательстве предыдущей теоремы используются только оценки для характеристических чисел, то она останется верной, если в условии 2) потребовать только ограниченность сверху главных характеристических чисел псевдоорграфов и псевдографов, принадлежащих множеству $\Sigma$.

Сопоставляя последнюю теорему с теоремами $1,3,4$, получаем следующие утверждения.

ТЕОРЕМа 6. Пусть $r, m$ - произвольные натуральные числа и $\Sigma$ - множество конечных попарно неизоморфных связных псевдографов, удовлетворяющ, следующим условиям:

1) степени всех вершин каждого $G \in \Sigma$ не превосходят числа $r$;

2) алгебраическая степень каждого $G \in \Sigma$ не больие числа $\mathrm{m}$. 
Тогда мнохество $\Sigma$ конечно.

Эта теорема помимо упомянутого вьше результата Цветковича [4] усиливает также основной результат [7].

ТЕОРема 7. Пусть $r, m$ - произвольные натуральные числа. Тогда множество арифметических длин любого мнохсества конечных сильно связных псевдоорграфов, алгебраическая степень кажсдого из которых равна $m$, а полустепени захода и исхода всех вершин - числу $r$, конечно.

СлЕДСТВИЕ. Пусть $r, m, h$ - произвольные натуральные числа $и \Sigma$ - множество конечных попарно неизоморфных сильно связных регулярных псевдоорграфов, удовлетворяющих следующим условиям:

1) полустепень каждого $G \in \Sigma$ не превосходит числа $r$;

2) алгебраическая степень каждого $G \in \Sigma$ не больше числа $m$;

3) арифметическая высота каждого $G \in \Sigma$ не больше числа $h$.

Тогда множество $\Sigma$ конечно.

ТЕОрема 8. Пусть $r, m$ - произвольные натуральные числа и $\Sigma$ - множество конечных попарно неизоморфных сильно связных вериинно-симметричных псевдоорграфов, удовлетворяюших следующим условиям:

1) полустепень каждого $G \in \Sigma$ не превосходит числа $r$;

2) алгебраическая степень каждого $G \in \Sigma$ не больше числа $\mathrm{m}$.

Тогда множество $\Sigma$ конечно.

Результаты настоящей работы анонсированы автором в [8].

\section{СПИСОК ЦИТИРОВАННОЙ ЛИТЕРАТУРЫ}

[1] Цветкович Д., Дуб М., Захс Х. Спектры графов. Теория и применение. Киев: Наукова думка, 1984.

[2] Кострикин А.И. Вокруг Бернсайда. М.: Наука, 1986.

[3] Харари $\Phi$. Теория графов. М.: Мир, 1973.

[4] Cvetković D. M. Cubic integral graphs // Univ. Beograd Publ. Elektrotehn. Fak., Ser. Mat. Fiz. 1975. № 498-541. P. 107-113.

[5] Гантмахер Ф.Р. Теория матриц. М.: Наука, 1966.

[6] Маркус М., Минк Х. Обзор по теории матриц и матричных неравенств. М.: Наука, 1972.

[7] Струнков С. П. Об одной теореме Д. Цветковича в теории графов // УМН. 1990. Т. 314. № 6. С. $145-146$.

[8] Струнков С.П. О слабо коспектральных графах // Докл. РАН. 2001. Т. 379. №3. C. 302-304.

Московский инженерно-физический институт (государственньй университет)

Поступило E-mail: strunkov@ium.ips.ras.ru 10.01 .2003

Исправленный вариант 28.05.2003 